\title{
Measuring sub-nanometre thickness changes during phase transitions of supported lipid bilayers with quantitative differential interference contrast microscopy
}

David Regan, Joseph Williams, Francesco Masia, Paola Borri, Wolfgang Langbein

David Regan, Joseph Williams, Francesco Masia, Paola Borri, Wolfgang Langbein, "Measuring sub-nanometre thickness changes during phase transitions of supported lipid bilayers with quantitative differential interference contrast microscopy," Proc. SPIE 10887, Quantitative Phase Imaging V, 1088719 (4 March 2019); doi: 10.1117/12.2510461

SPIE. Event: SPIE BiOS, 2019, San Francisco, California, United States 


\title{
Measuring sub-nanometre thickness changes during phase transitions of supported lipid bilayers with quantitative differential interference contrast microscopy
}

\author{
David Regan ${ }^{\mathrm{a}}$, Joseph Williams ${ }^{\mathrm{b}}$, Francesco Masia ${ }^{\mathrm{a}, \mathrm{b}}$, Paola Borri ${ }^{\mathrm{b}}$, and Wolfgang Langbein ${ }^{\mathrm{a}}$ \\ aSchool of Physics and Astronomy, Cardiff University, The Parade, Cardiff CF24 3AA, United \\ Kingdom

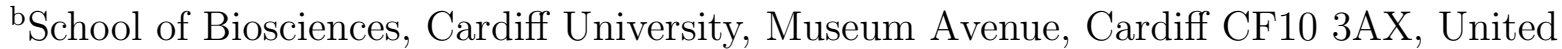 \\ Kingdom
}

\begin{abstract}
Quantitative differential interference contrast (qDIC) microscopy is applied to the study of the main phase transition of dipentadecanoylphosphatidylcholine $\left(\mathrm{DC}_{15} \mathrm{PC}\right)$ supported lipid bilayers. We measure thickness changes of about $1 \mathrm{~nm}$ occurring in the bilayer with sub-nanometre resolution and show how the presence of fluorescently labelled lipids, even at small concentrations, can broaden the phase transition.
\end{abstract}

Keywords: Supported lipid bilayer, phase transition, differential interference contrast

\section{INTRODUCTION}

It is now widely accepted that the cell plasma membrane is made up of different, coexisting, membrane phases. ${ }^{1,2}$ While the majority of the plasma membrane exists in the liquid disordered $\left(\mathrm{L}_{\mathrm{d}}\right)$ phase, ordered phases such as the cholesterol-induced liquid ordered $\left(\mathrm{L}_{\mathrm{o}}\right)$ phase are believed to exist as transient nanometre scale rafts which segregate membrane components, ${ }^{2}$ and have roles in viral infection, ${ }^{3}$ immune system signalling ${ }^{2}$ and lipid trafficking ${ }^{1}$ among other cellular processes.

Possibly the simplest model for the phase behaviour of the cell membrane is found in single component model membranes, during the liquid-to-solid phase transition which occurs when the lipid bilayer is cooled below the freezing point of the membrane lipid. ${ }^{1}$ In the solid ordered $\left(\mathrm{S}_{\mathrm{o}}\right)$ phase, the lipids are condensed with the acyl chains in an extended configuration reminiscent of the $\mathrm{L}_{\mathrm{o}}$ phase. This transition is usually studied experimentally using supported lipid bilayers (SLBs), planar lipid bilayers formed on a hydrophilic solid support, such as glass or mica. ${ }^{2,4}$

The transition can be visualised by the use of fluorescent labels. During the phase coexistence region of the transition, both $\mathrm{L}_{\mathrm{d}}$ and $\mathrm{S}_{\mathrm{o}}$ phases exist side by side. ${ }^{4}$ By incorporating into the bilayer fluorescent labels that selectively partition into one of the two phases (most fluorophores will partition into the $\mathrm{L}_{\mathrm{d}}$ phase) the different phases can be distinguished by differences in their fluorescence signal. ${ }^{2,3}$ While the use of fluorophores is straightforward and enables investigation of key parameters such as diffusion ${ }^{4}$ and local membrane environment, ${ }^{1}$ it cannot be assumed that the fluorophore itself doesn't affect the system under investigation. Important properties such as bilayer thickness, diffusion and stability are known to be affected by commonly used fluorophores at sufficiently high concentrations. ${ }^{5}$

A widely used alternative to the use of fluorescent labels to study the liquid-to-solid phase transition in SLBs is atomic force microscopy (AFM). ${ }^{4}$ As the lipid $\mathrm{S}_{\mathrm{o}}$ phase is thicker than the $\mathrm{L}_{\mathrm{d}}$ phase,${ }^{4}$ the $\mathrm{S}_{\mathrm{o}}$ phase regions are visible in the topographic maps of the SLB that AFM produces. AFM is limited however by the fact that it cannot distinguish between the contribution to the sample height of the SLB and its underlying hydration layer, and the difficulties in combining AFM with fluorescence.

Further author information: (Send correspondence to David Regan)

David Regan: E-mail: ReganDC@cardiff.ac.uk 
Quantitative differential interference contrast (qDIC) is a technique that uses Wiener filtering to reconstruct the phase profile of a sample from standard differential interference contrast (DIC) images. As qDIC images are captured using a conventional microscope setup, qDIC can be used in conjunction with fluorescence imaging to obtain complimentary sample information. This technique has already been used to measure the lamellarity of giant unilamellar vesicles (GUVs) ${ }^{6}$ and to measure changes in SLB thickness resulting from the preparation conditions of the support. ${ }^{7}$ The greater thickness and refractive index of the $\mathrm{S}_{\mathrm{o}}$ phase relative to the surrounding $\mathrm{L}_{\mathrm{d}}$ phase creates optical thickness gradients at the phase boundary, which enable the reconstruction of the optical thickness difference between the two phases in the qDIC image.

Here, we show how qDIC can be used to observe the liquid-to-solid phase transition in SLBs, and obtain information on changes in bilayer thickness with sub-nanometre precision. We also investigate how the inclusion of a typical fluorophore label perturbs the phase transition. This has previously been investigated for fluorophores attached to the phospholipid acyl chains, ${ }^{8}$ but comparatively little attention has been paid to the effect of head-labelled fluorophores. To this end, we investigate the influence of the ATTO488 attached to the dioleoylphosphatidylethanolamine head group (ATTO488-DOPE) on the liquid-to-solid phase transition.

\section{MATERIALS \& METHODS}

Supported lipid bilayers were prepared by spin coating, using a procedure based on that developed by Mennicke and Salditt. ${ }^{9}$ Glass surfaces were cleaned first by gentle wiping with acetone soaked lens paper, followed by piranha etching, in which glass coverslips were immersed in a 3:1 volumetric ratio of sulphuric acid and hydrogen peroxide (30\% in water) at $95{ }^{\circ} \mathrm{C}$ for one hour. The piranha etching process also renders the glass hydrophilic, which encourages bilayer formation. The glass was then fully wetted with $150 \mathrm{\mu l}$ of lipid mixture in 2-propanol solvent, and the coverslip rotated about its centre axis at $3600 \mathrm{rpm}$ for 30 seconds, with 6 second acceleration and deceleration stages. The coverslip was incubated in a humidified nitrogen environment at $37^{\circ} \mathrm{C}$ for one hour before being sealed onto a glass slide using a Grace Bio-Labs (Bend, US) SecureSeal imaging spacer. The interior was filled with a pH 7.4 phosphate buffered saline $(\mathrm{PBS})$ solution at $1 \times$ concentration from Gibco (Gaithersburg, US), which was degassed in vacuum for five minutes before use to remove small air bubbles which were visible on the sample in DIC.

Lipid solutions were prepared from mixtures of 1,2-dipentadecanoyl-sn-glycero-3-phosphocholine $\left(\mathrm{DC}_{15} \mathrm{PC}\right)$ and 1,2-dioleoyl-sn-glycero-3-phosphoethanolamine with ATTO488 tagged to the lipid headgroup (ATTO488DOPE). Lipids were purchased in powered form from Avanti Polar Lipids (Alabaster, US) and ATTO-TEC (Siegen, Germany) respectively, and used without further purification. The extent of lipid coverage on the surface was controlled by adjusting the concentration of the lipids in solution. Typically, concentrations ranged from $0.8 \mathrm{mg} / \mathrm{ml}$ to $1.2 \mathrm{mg} / \mathrm{ml}$ in order to achieve a layer one bilayer thick with enough defects to allow measurement of the phase step over the bilayer edge. The 2-propanol solvent was HPLC grade purchased from Sigma-Aldrich (St. Louis, US).

Images were taken using a CCD camera (Hamamatsu Orca 285, with $1344 \times 1024$ pixels of $6.45 \mu \mathrm{m}$ size, $18 \mathrm{ke}$ full well capacity, and 7 e read noise) on a Nikon Eclipse Ti-U inverted microscope. All images used a $20 \times 0.75$ NA dry objective with a $1.5 \times$ tube lens for an overall magnification of 30 . Differential interference contrast (DIC) images used a $100 \mathrm{~W}$ halogen lamp, filtered using a Nikon GIF (green interference filter, centre wavelength 550 $\mathrm{nm}, 70 \mathrm{~nm}$ full width at half maximum) and a Schott BG40 filter (to remove infra-red wavelengths outside the operating range of the DIC polarisers). The shear distance on the sample using Nikon N2 prisms was measured ${ }^{6}$ to be $(238 \pm 10) \mathrm{nm}$. Fluorescence images used a Prior Lumen 200 lamp with a Semrock GFP-A-BASIC-000 filter cube for illumination, attenuated as necessary using Nikon ND4 and ND8 filters.

To reduce image noise, DIC images were averaged over 100 frames with $100 \mathrm{~ms}$ exposure time each. To eliminate the effect of inhomogeneous illumination and sensor sensitivity, pairs of DIC images $I_{ \pm}$were taken for each field of view and time point, at opposite polariser angles, either $\pm 12.9^{\circ}$ or $\pm 15.0^{\circ}$. The two images were then combined to a contrast image $I_{\mathrm{C}}=\left(I_{+}-I_{-}\right) /\left(I_{+}+I_{-}\right)$, which was then converted into a qDIC phase image using the procedure described in Ref.10,11. 
$\mathbf{a}$

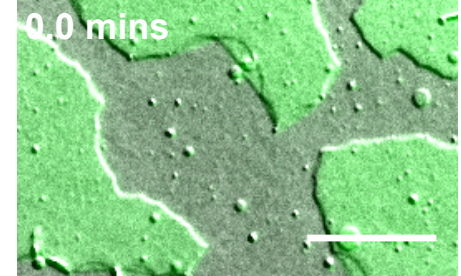

d

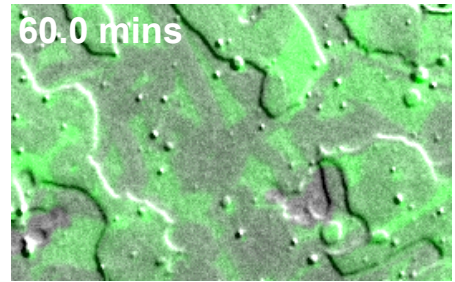

b

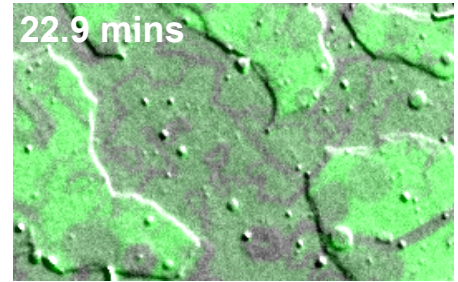

e

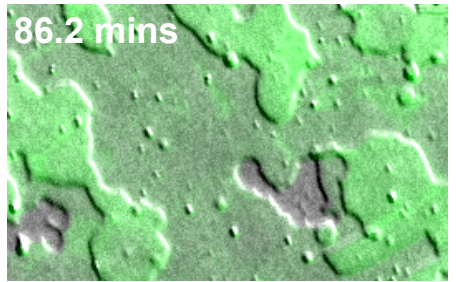

C

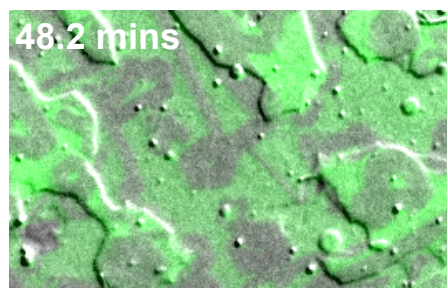

f

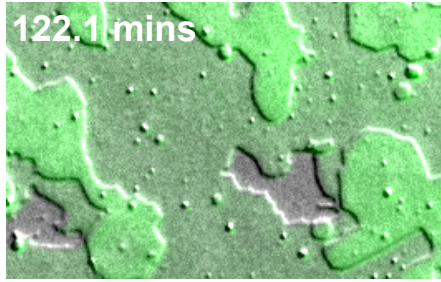

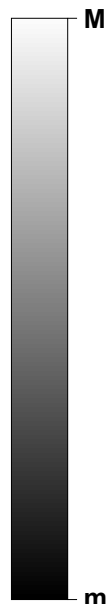

Figure 1. qDIC contrast images of a region of a $\mathrm{DC}_{15} \mathrm{PC}$ bilayer labelled with 1 mol\% ATTO488-DOPE undergoing a phase transition during cooling with fluorescence intensity as overlay in the green colour channel, showing the accumulation of fluorophore in the shrinking $\mathrm{L}_{\mathrm{d}}$ phase regions. The images show the region at set temperatures of a) $\left.33.9^{\circ} \mathrm{C}, \mathrm{b}\right) 33.3^{\circ} \mathrm{C}$, c) $32.7^{\circ} \mathrm{C}$, d) $32.4^{\circ} \mathrm{C}$, e) $31.8^{\circ} \mathrm{C}$, f) $30.9^{\circ} \mathrm{C}$. The time elapsed from the acquisition of image a) is shown in the images. The $\mathrm{qDIC}$ contrast is scaled from $\mathrm{m}=-0.002$ to $\mathrm{M}=0.001$.

\section{RESULTS \& DISCUSSION}

To investigate the thickness changes during the main phase transition, we cooled SLBs prepared from the saturated chain lipid $\mathrm{DC}_{15} \mathrm{PC}$ from above to below its nominal phase transition temperature ${ }^{12}$ of $33^{\circ} \mathrm{C}$. The samples were cooled in $0.3{ }^{\circ} \mathrm{C}$ steps, each followed by a 10 minute equilibration time and approximately 3 minutes of imaging, resulting in an average cooling rate of $1.4^{\circ} \mathrm{C} /$ hour. At temperatures close to the phase transition, $\mathrm{L}_{\mathrm{d}}$ and $\mathrm{S}_{\mathrm{o}}$ phase regions coexist, ${ }^{4,12}$ which we measure using qDIC. In SLBs prepared by spin coating, the coverage of the glass can vary, with some regions being two or more bilayers thick. Due to the inherent difficulty in interpreting the phase behaviour in stacks of multiple bilayers, we used here exclusively the single bilayer regions for the analysis of the phase transition.

Such phase transitions are affected by the presence of impurities, as they are excluded from the solid phase, and thus reduce the phase transition temperature, similar to the effect of salt lowering the freezing point of water. The fluorescently labelled lipids represent such an impurity, and are thus expected to influence the phase transition. We therefore measured the phase transition for different concentrations of ATTO488-DOPE, which is excluded from the frozen $\mathrm{S}_{\mathrm{o}}$ domains, and accumulates in the $\mathrm{L}_{\mathrm{d}}$ domains, progressively reducing their freezing temperature. This accumulation is clearly visible in the images given in Fig. 1.

To measure the fluorophore concentration, we use the fluorescence intensity, normalised to the initial homogeneous liquid phase, and corrected for photobleaching. The fluorophore concentration in the $\mathrm{L}_{\mathrm{d}}$ domains increases as their volume fraction decreases while cooling. Assuming the fluorescence intensity scales linearly with the fluorophore concentration, we show the concentration as function of temperature in Fig. 2 for bilayers containing either 0.01 or $1.00 \mathrm{~mol} \%$ ATTO488-DOPE. In the latter sample, the fluorophore concentration in the $\mathrm{L}_{\mathrm{d}}$ phase reaches $2 \mathrm{~mol} \%$ before it finally freezes at a temperature $2.7^{\circ} \mathrm{C}$ below the point at which $\mathrm{S}_{\mathrm{o}}$ phase domains first appeared. As the $\mathrm{S}_{\mathrm{o}}$ domains form, the concentration of fluorophore within them increases gradually over the course of the phase transition, from a starting concentration of $0.35 \mathrm{~mol} \%$, eventually reaching levels apparently higher that the starting concentration once the bilayer is in a single phase. This is attributed to the lateral contraction of the bilayer when freezing, increasing the areal density of fluorophore. Once frozen, the fluorophore diffuses slowly in the solid bilayer, establishing an equilibrium over a timescale of several hours at room temperature.

When the overall proportion of fluorophore is reduced to $0.01 \mathrm{~mol} \%$, the range of temperatures over which phase coexistence is visible is reduced to $0.6{ }^{\circ} \mathrm{C}$, due to there being less fluorophore to lower the phase transition temperature in the $\mathrm{L}_{\mathrm{d}}$ phase; the fluorophore concentration in the $\mathrm{L}_{\mathrm{o}}$ phase peaks at 3.6 times the starting 


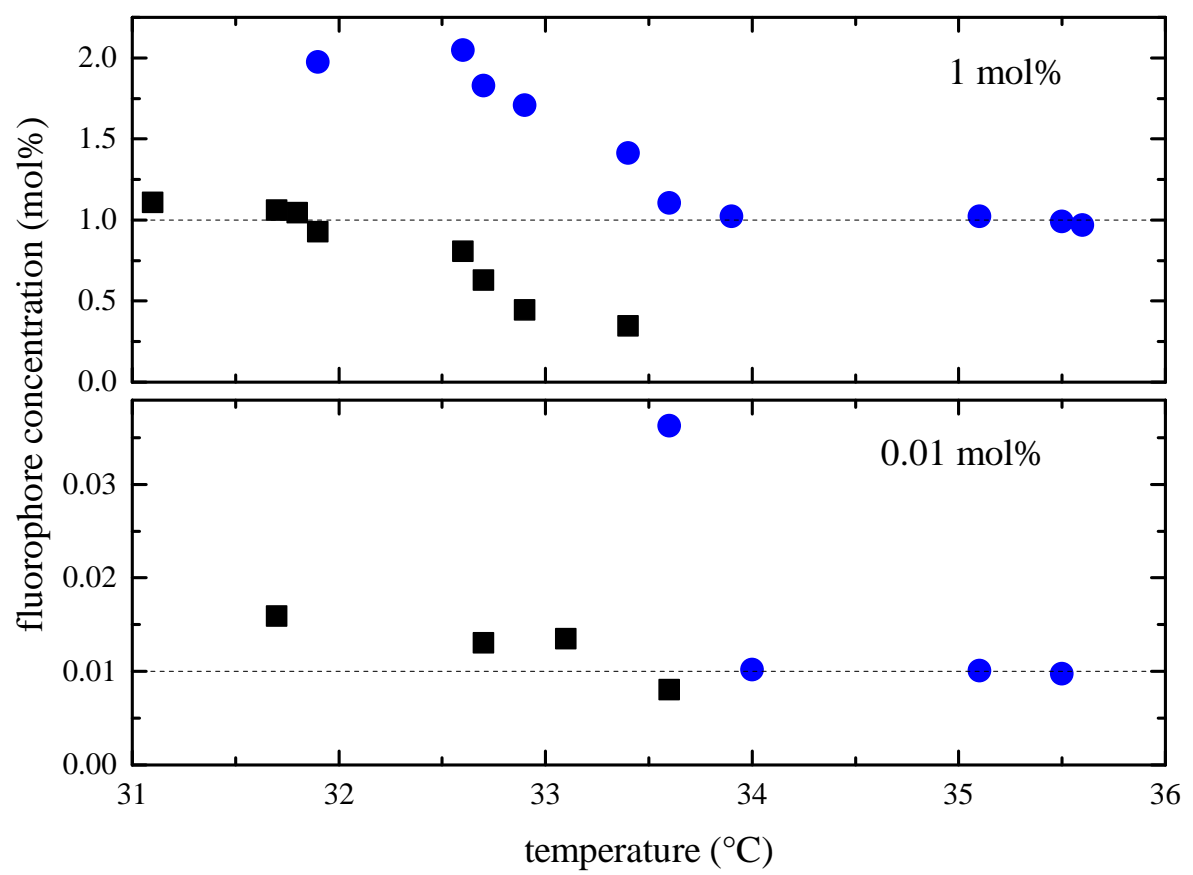

Figure 2. Fluorophore concentration (calculated from the bleach corrected fluorescence intensity) in both the fluid (circles) and gel (squares) phases against temperature during the phase transition of the $\mathrm{L}_{\mathrm{d}}$ phase of a $\mathrm{DC}_{15} \mathrm{PC}$ bilayer. The top panel shows data from a bilayer prepared using a fluorophore concentration of $1.00 \mathrm{~mol} \%$, while the lower panel shows data for a bilayer prepared using $0.01 \mathrm{~mol} \%$.

concentration. Using qDIC we can also observe the phase transition without the need for fluorophore. When no fluorescent labels are incorporated into the bilayer, phase coexistence was visible over a $0.6{ }^{\circ} \mathrm{C}$ range, with some variation across the field of view. We attribute this observation to the interaction with the substrate, which may vary across the sample. This suggests that when the fluorophore concentration is reduced to $0.01 \mathrm{~mol} \%$, the behaviour of the SLB is effectively the same as that of an unlabelled SLB, with a finite coexistence region due to the interaction with the support, or the finite purity of the $\mathrm{DC}_{15} \mathrm{PC}$ used.

Since the fluorophore is not needed to visualise the phase transition using qDIC, we repeated the experiment using unlabelled $1 \mathrm{~mol} \%$ DOPE instead of ATTO488-DOPE. We found a phase coexistence range of at least 2.1 ${ }^{\circ} \mathrm{C}$. This is comparable to that measured with ATTO488-DOPE as the impurity. This suggests that the cause of the elongation of the phase transition is the difference in the hydrocarbon tails, which are unsaturated of the DOPE molecule, rather than the prescence opf the ATTO488 fluorophore. This might be expected, as the fluorophore on the head group sticks out of the bilayer, and thus is unlikely to affect the bilayer ordering during freezing.

By measuring the step in optical thickness over the phase boundary, the absolute thickness difference between coexisting $\mathrm{S}_{\mathrm{o}}$ and $\mathrm{L}_{\mathrm{d}}$ phases can be measured. ${ }^{7}$ Fig. 3 shows the appearance of an $\mathrm{L}_{\mathrm{d}}$ domain surrounded by an expanding $\mathrm{S}_{\mathrm{o}}$ domain during the phase transition. For this analysis, a refractive index of 1.44 was assumed for both the gel and fluid phases of $\mathrm{DC}_{15} \mathrm{PC}$. This value is derived from reported average refractive indices of two structurally similar lipids, $\mathrm{DMPC}^{13}$ (1.44) and $\mathrm{DPPC}^{14}$ (1.4381). While the refractive index is affected by temperature, exhibiting a sharp change around the phase transition, we consider this to be negligible here as the change is smaller than the uncertainty on our refractive index estimate. ${ }^{15} \mathrm{~A}$ thickness difference between coexisting gel and fluid phases of $1.00 \pm 0.07 \mathrm{~nm}$ was measured at $32.0^{\circ} \mathrm{C}$ in the first bilayer. This is consistent with the height difference between gel and fluid phase regions of a DPPC supported bilayer during heating measured using AFM. ${ }^{16}$

The absolute thicknesses of each phase was also measured separately during the cooling of a $\mathrm{DC}_{15} \mathrm{PC}$ :ATTO488-DOPE (99.9:0.1) bilayer at the same rate described before. The thickness of each of the bilayer 
a

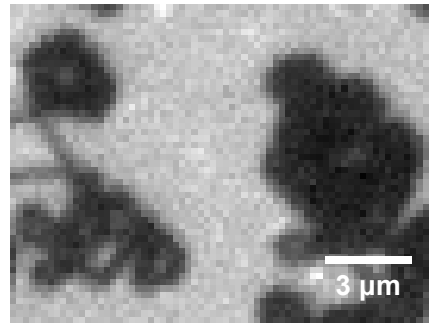

b

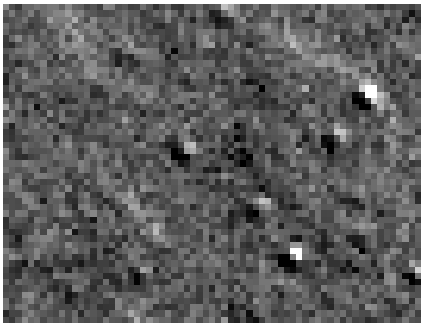

C

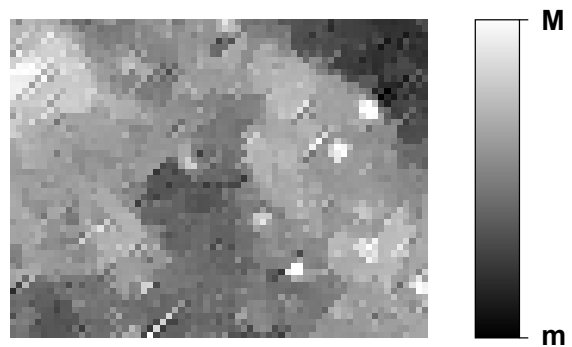

Figure 3. A region containing both $\mathrm{S}_{\mathrm{o}}$ and $\mathrm{L}_{\mathrm{d}}$ domains at $32.8^{\circ} \mathrm{C}$, shown in a) fluorescence $(M=280 \mathrm{pe}, m=40 \mathrm{pe})$, b) qDIC contrast $(m=-0.0007, M=+0.0014)$, and c) qDIC phase $(m=-2.7 \mathrm{mrad}, M=+1.2 \mathrm{mrad})$. Ordered domains appear as dark regions in the fluorescence image and lighter regions in the phase image.

phases at different temperatures are shown in Fig. 4, where the sharp difference in the thickness between the $\mathrm{S}_{\mathrm{o}}$ and $\mathrm{L}_{\mathrm{d}}$ phases is visible. While the thickness of the $\mathrm{L}_{\mathrm{d}}$ phase appears to gradually increase as the temperature decreases in the phase coexistence region, consistent with measurements on similar bilayers with X-ray and neutron scattering, ${ }^{17}$ the thickness of the $\mathrm{S}_{\mathrm{o}}$ phase remains constant within error. At $35.6^{\circ} \mathrm{C}$, above the bilayer phase transition temperature, a thickness of $3.81 \pm 0.07 \mathrm{~nm}$ was measured for the first bilayer, while below the phase transition temperature, at $26.9^{\circ} \mathrm{C}$, the first bilayer thickness was $4.91 \pm 0.05 \mathrm{~nm}$. The difference in thickness between the two measurements is $1.10 \pm 0.08 \mathrm{~nm}$, close to that measured in the phase coexistence region.

There are few direct measurements of the absolute thickness of $\mathrm{DC}_{15} \mathrm{PC}$ bilayers in the literature. Surface topography measurements on $\mathrm{DC}_{15}$ PC bilayers formed on mica in PBS solution give an $\mathrm{S}_{\mathrm{o}}$ phase thickness of 4.9 $\pm 0.2 \mathrm{~nm} .{ }^{18}$ Given that AFM measures the combined bilayer and hydration layer thickness while we measure the bilayer thickness only, it would be expected that our values be smaller than measured using AFM by an amount equal to the thickness of the hydration layer between the bilayer and the support. That our measurement is very close to the AFM measurement may be due to indentation of the bilayer by the AFM tip ${ }^{18}$ or the absence of the hydration layer.

Previous experiments on supported bilayers formed on hydrophilic mica surfaces have shown that the influence of the substrate on the proximal leaflet of the bilayer can result the two leaflets undergoing phase transitions at different temperatures. ${ }^{4,12}$ The height difference we measure between coexisting $\mathrm{L}_{\mathrm{d}}$ and $\mathrm{S}_{\mathrm{o}}$ phases in $\mathrm{DC}_{15} \mathrm{PC}$

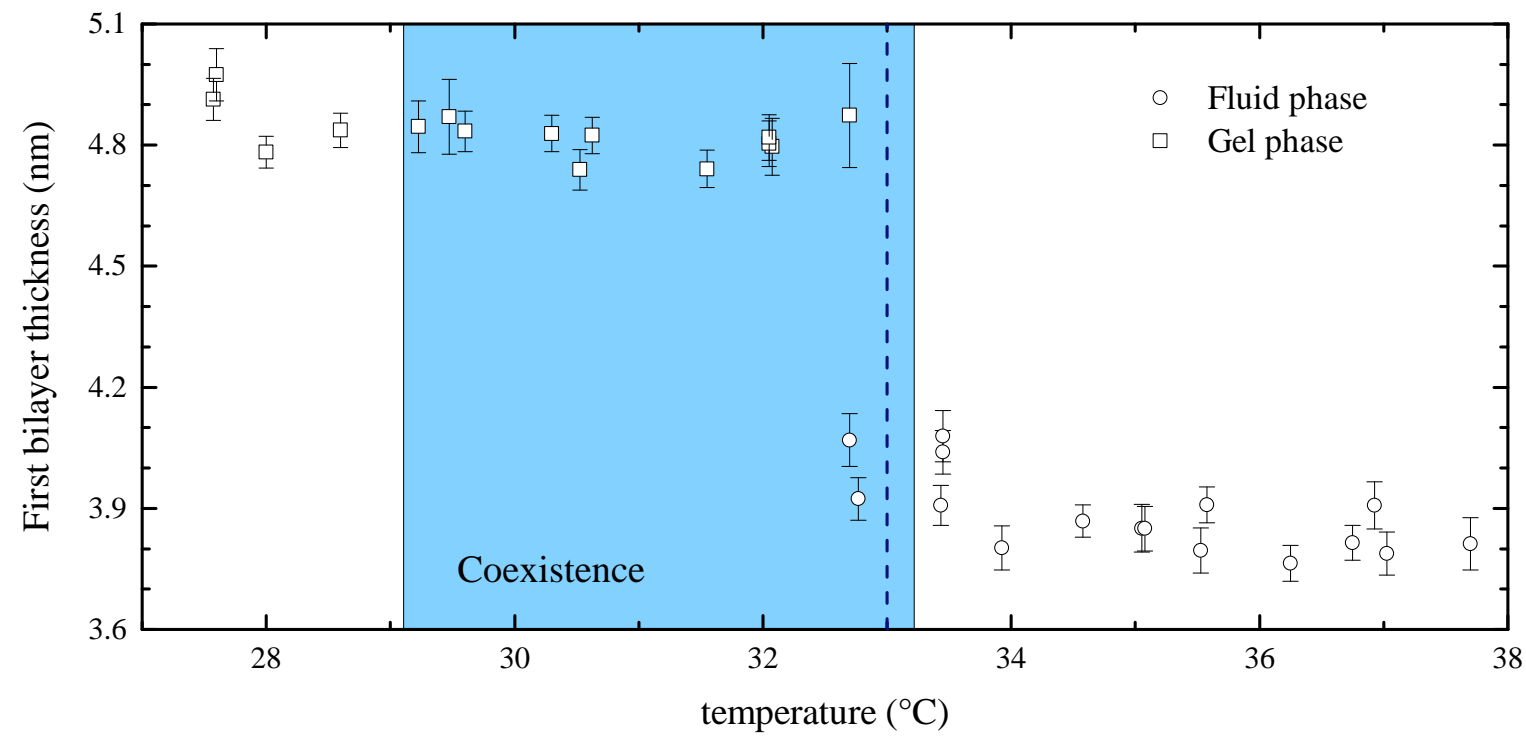

Figure 4. The thickness of $\mathrm{S}_{\mathrm{o}}$ and $\mathrm{L}_{\mathrm{d}}$ regions of a $\mathrm{DC}_{15} \mathrm{PC}$ bilayer versus temperature measured using qDIC. The nominal phase transition temperature ${ }^{12}$ of $\mathrm{DC}_{15} \mathrm{PC}$ of $33^{\circ} \mathrm{C}$ is denoted by a vertical dashed line. The region in which phase coexistence is visible in fluorescence is coloured blue. 
bilayers suggests that the phase transitions in the two leaflets remain coupled in our samples; that is, the $\mathrm{S}_{\mathrm{o}}$ phase begins to form at the same temperature in both leaflets, and the phase edges overlap exactly. This is consistent with previous measurements on glass surfaces. ${ }^{19}$

However, by looking at regions of the lipid film that are two bilayers thick, a slight separation appears to be present in the phase transition temperature of the first bilayer, in close proximity to the support, and the second bilayer, formed on top of the first and so separated from the influence of the support altogether. While $\mathrm{S}_{\mathrm{o}}$ domains first appeared in the same frame for both the first and second bilayers, the $\mathrm{S}_{\mathrm{o}}$ domains in the first bilayer were always larger and more numerous than those in the second bilayer. This indicates that the phase transition temperature has increased for the bilayer closest to the support; this is an established effect of the support on the phase transition. ${ }^{4}$ The fact that phase coexistence appears in the same frame would mean that the temperature separation of the phase transition temperature of the two bilayers would have to be smaller than the temperature interval between subsequent images.

\section{CONCLUSION}

Quantitative DIC was used to track changes in SLBs over a liquid to solid phase transition, enabling the measurement of changes in bilayer thickness as the sample was cooled, and to visualise coexisting $\mathrm{S}_{\mathrm{o}}$ and $\mathrm{L}_{\mathrm{d}}$ phases in a $\mathrm{DC}_{15} \mathrm{PC}$ bilayer. The measured SLB thicknesses of the two phases are larger than expected from comparisons with AFM measurements which measure the sum of the bilayer and hydration layer thickness. The height difference between coexisting $L_{d}$ and $S_{o}$ phases is $1.00 \pm 0.07 \mathrm{~nm}$, consistent with AFM measurements on bilayers formed from similar lipids.

Notably, we observe an extension of the phase transition down to $3^{\circ} \mathrm{C}$ below the nominal temperature for a fluorescence labelling density of $1 \mathrm{~mol} \%$, showing a melting point reduction by the presence of the labelled lipid which accumulates in the contracting $\mathrm{L}_{\mathrm{d}}$ phase regions. This seems to be the result of the DOPE lipid to which the fluorophore is attached, rather than the fluorophore itself affecting the phase transition, as unlabelled DOPE was similarly affecting the phase transition. We note that this comparison is enabled by observing the phase transition without fluorescence labelling using qDIC. It would be expected therefore that attaching the ATTO fluorophore to lipids with a phase transition temperature closer to that of $\mathrm{DC}_{15} \mathrm{PC}$ such as $\mathrm{DPPE}$ would reduce the perturbative effect of fluorescence labelling on the phase transition. Such a matching is, however, costly, as it will often require custom labelling.

Consistent with previous results we find that in stacks of multiple bilayers, the bilayer in closest proximity to the hydrophilic glass support transitions more rapidly, showing that the phase transition is also affected by the support. However, the data also shows that the two leaflets within this first bilayer undergo the phase transition together, showing that any effect of the glass on the bilayer is not sufficient to trigger decoupled phase transitions such as those observed on mica.

The data presented in this work are available from the Cardiff University data archive, under the digital object identifier 10.17035/d.2019.0067230970.

\section{ACKNOWLEDGMENTS}

The microscope setup used was developed within the UK BBSRC Research Council (grant n. BB/H006575/1). D.R. and J.W. acknowledge funding by an EPSRC DTA studentship (EP/M507842/1). P.B. acknowledges the UK EPSRC Research Council for her Leadership fellowship award (EP/I005072/1). W.L. acknowledges support by his Leverhulme Royal Society Research Fellowship (LT20085). The authors thank Iestyn Pope for assistance in the data acquisition, and George Zorinyants for contributions to the qDIC analysis software.

\section{REFERENCES}

[1] M'Baye, G., Mély, Y., Duportail, G., and Klymchenko, A. S., "Liquid ordered and gel phases of lipid bilayers: Fluorescent probes reveal close fluidity but different hydration," Biophys. J. 95(3), 1217 - 1225 (2008). 
[2] Sezgin, E., Levental, I., Mayor, S., and Eggeling, C., "The mystery of membrane organization: composition, regulation and roles of lipid rafts," Nat. Rev. Mol. Cell Biol. 18(6), 361-374 (2017).

[3] Yang, S.-T., Kiessling, V., and Tamm, L. K., "Line tension at lipid phase boundaries as driving force for HIV fusion peptide-mediated fusion," Nat. Commun. 7(11401), 1-9 (2016).

[4] Alessandrini, A. and Facci, P., "Phase transitions in supported lipid bilayers studied by AFM," Soft Matter 10, 7145-7164 (2014).

[5] Flynn, K. R., Sutti, A., Martin, L. L., Ackland, M. L., and Torriero, A. A., "Critical effects of polar fluorescent probes on the interaction of DHA with POPC supported lipid bilayers," Biochim. Biophys. Acta, Biomembr. 1860(5), 1135 - 1142 (2018).

[6] McPhee, C. I., Zoriniants, G., Langbein, W., and Borri, P., "Measuring the lamellarity of giant lipid vesicles with differential interference contrast microscopy," Biophys. J. 105(6), 1414 - 1420 (2013).

[7] Regan, D., Williams, J., Borri, P., and Langbein, W., "Lipid bilayer thickness measured by quantitative DIC reveals phase transitions and effects of substrate hydrophilicity," submitted (2019).

[8] Loura, L. M., Fernandes, F., Fernandes, A., and Ramalho, J. P., "Effects of fluorescent probe NBD-PC on the structure, dynamics and phase transition of DPPC. a molecular dynamics and differential scanning calorimetry study," Biochim. Biophys. Acta, Biomembr. 1778(2), 491 - 501 (2008).

[9] Mennicke, U. and Salditt, T., "Preparation of solid-supported lipid bilayers by spin-coating," Langmuir 18(21), 8172-8177 (2002).

[10] Pope, I., Payne, L., Zoriniants, G., Thomas, E., Williams, O., PeterWatson, Langbein, W., and Borri, P., "Coherent anti-stokes raman scattering microscopy of single nanodiamonds," Nat. Nanotechnol. 9, 940-946 (2014).

[11] Williams, J. B., Regan, D., Borri, P., and Langbein, W. in preparation (2019).

[12] Feng, Z. V., Spurlin, T. A., and Gewirth, A. A., "Direct visualization of asymmetric behavior in supported lipid bilayers at the gel-fluid phase transition," Biophys. J. 88(3), $2154-2164$ (2005).

[13] Howland, M. C., Szmodis, A. W., Sanii, B., and Parikh, A. N., "Characterization of physical properties of supported phospholipid membranes using imaging ellipsometry at optical wavelengths," Biophys. J. 92(4), $1306-1317$ (2007).

[14] Wang, M., Liu, C., Thormann, E., and Dédinaitè, A., "Hyaluronan and phospholipid association in biolubrication," Biomacromolecules 14(12), 4198-4206 (2013).

[15] Horváth, R., Fricsovszky, G., and Papp, E., "Application of the optical waveguide lightmode spectroscopy to monitor lipid bilayer phase transition," Biosens. Bioelectron. 18(4), 415 - 428 (2003).

[16] Leonenko, Z., Finot, E., Ma, H., Dahms, T. S., and Cramb, D., "Investigation of temperature-induced phase transitions in DOPC and DPPC phospholipid bilayers using temperature-controlled scanning force microscopy," Biophys. J. 86(6), 3783 - 3793 (2004).

[17] Kučerka, N., Nieh, M.-P., and Katsaras, J., "Fluid phase lipid areas and bilayer thicknesses of commonly used phosphatidylcholines as a function of temperature," Biochim. Biophys. Acta, Biomembr. 1808(11), $2761-2771$ (2011).

[18] Beckmann, M., Nollert, P., and Kolb, H.-A., "Manipulation and molecular resolution of a phosphatidylcholine-supported planar bilayer by atomic force microscopy," J. Membr. Biol. 161(3), 227-233 (1998).

[19] Harb, F. F. and Tinland, B., "Effect of ionic strength on dynamics of supported phosphatidylcholine lipid bilayer revealed by FRAPP and Langmuir-Blodgett transfer ratios," Langmuir 29(18), 5540-5546 (2013). 